\title{
The category effect in visual selective attention
}

\author{
PATTI L. KELLY and DAVID W. HARRISON \\ Virginia Polytechnic Institute and State University, Blacksburg, Virginia \\ and \\ MILTON H. HODGE \\ University of Georgia, Athens, Georgia
}

\begin{abstract}
The effects of extended practice on the development of the category effect were assessed in a two-part study. Four groups were trained on within- and between-category search, using a detection task similar to that used by Jonides and Gleitman (1972). These groups then received extended testing requiring both detection and identification of opposite-category items. One, two, and three target-category items and a variable number of opposite-category items were presented on each trial. Evidence was found for partially selective processing based on category membership. Both the number of within-category items and the number of between-category distractors influenced response latency. The results indicate that practice as well as the density of items within the visual array is critical to the development of the category distinction.
\end{abstract}

The quality and amount of practice provided in a search task are thought to be critical variables in the development of automatic detection, as has been proposed by Shiffrin, Schneider, and their colleagues (Schneider \& Shiffrin, 1977; Shiffrin, Dumais, \& Schneider, 1981; Shiffrin \& Schneider, 1977). Yet despite the welldocumented role of practice in improving performance in tasks that involve detection and classification, little attention has been paid specifically to the role of practice in the development of the category effect.

The present research was conducted to examine the effect of extended practice on the development of the category effect. A further goal of the study was to determine whether the category effect is a form of automatic detection (Shiffrin \& Schneider, 1977; Shiffrin et al., 1981).

\section{METHOD}

The experiment was conducted in two distinct parts: a training phase and a testing phase. Table 1 presents an overview of the experimental design.

\section{Subjects}

Nineteen men and 21 women undergraduates participated. Subjects were screened using the Master Orthorater to ensure a minimum visual acuity of 20/30 in both eyes. Subjects wearing eyeglasses or hard contact lenses were excluded from participation.

\section{Stimulus Materials}

The stimuli were constructed with Prestype 36-point black Futura Demi uppercase letters, photographed, and mounted in $2 \times 2$ in. slides. The stimulus slides were displayed in a conventional three-channel tachistoscope at a viewing distance of $96 \mathrm{~cm}$. Luminances in all channels (including a fixation channel) were set at $206 \mathrm{~cd} / \mathrm{m}^{2}$. The number of stimuli per slide varied from one to six. To maintain a constant overall visual

Address correspondence to David W. Harrison, Department of Psychology, Virginia Polytechnic Institute and State University, Blacksburg, VA 24061-0436. angle, each stimulus was located at one to eight equally spaced locations on the circumference of an imaginary circle.

When only one element was present in the display, it occurred at any of the eight locations. When two elements were presented, they were located at diametrically opposed loci. When three elements were present, two were diametrically opposed, and the third was randomly positioned among the remaining loci. Similar procedures were used to construct arrays with four, five, and six elements. The center-to-center distance between two diametrically opposed elements subtended a visual angle of $3^{\circ}$; the minimum visual angle between the edges of the two adjacent elements was $.81^{\circ}$. The stimulus elements themselves were $.4^{\circ}$ in height, with a stroke width of $.068^{\circ}$.

The uppercase letters A, B, C, D, E, F, G, J, K, L, P, R, T, U, V, and $Y$ were used as both targets and distractors. For two groups of subjects (Groups RR and RU), these letters were rotated $180^{\circ}$ on the horizontal axis, appearing "upside-down and backwards." Each letter and all possible array locations were used about equally often. On any particular slide, no letter occurred more than once, regardless of its orientation.

\section{Design and Procedure}

A four-factor mixed design was used with the following factors:

Group. Subjects were randomly assigned to four groups (UU, UR, $R R$, and RU). Groups UU and UR received slides in which the target letter was always upright on the horizontal axis. During Training Block 1 (within-category search), these groups were required to detect a particular upright letter from among distractor letters that were also upright in orientation. During Training Block 2 (between-category search), the target letters remained upright, but all distractor letters were rotated $180^{\circ}$ on the horizontal axis from the target. The subject's task was to detect the presence of the between-category item (target letter) of upright orientation from among rotated letters. During training, Groups $\mathbf{R R}$ and $R U$ received slides in which the target letters were always rotated $180^{\circ}$ on the horizontal axis, giving them the appearance of being "upside-down and backwards." During within-category search (Training Block 1), these subjects were required to detect a particular rotated letter among other rotated letters. During between-category search (Training Block 2), these subjects were required to detect the presence of a rotated letter among unrotated letters.

During the testing phase of the study, Groups UU and RR continued to search for targets of the same orientation as that which they had used during training. Groups UR and RU, however, were switched to targets of the opposite category from training.

Blocks. During the training phase of the study, the blocking factor determined which type of search was required of the subject. During 
Table 1

Overview of the Experimental Design

\begin{tabular}{|c|c|c|c|c|c|}
\hline \multirow{2}{*}{$\begin{array}{l}\text { Subject } \\
\text { Group }\end{array}$} & \multicolumn{2}{|c|}{$\begin{array}{c}\text { Training } \\
\text { (Detection Task) }\end{array}$} & \multicolumn{3}{|c|}{$\begin{array}{c}\text { Test } \\
\text { (Identification Task) }\end{array}$} \\
\hline & Block 1 & Block 2 & Block 1 & Block 2 & Block 3 \\
\hline RR & $\begin{array}{l}\text { Within-category search } \\
\text { for rotated letter }\end{array}$ & $\begin{array}{l}\text { Between-category search } \\
\text { for rotated letter }\end{array}$ & 1 rotated letter & 2 rotated letters & 3 rotated letters \\
\hline RU & Within-category search & Between-category search & 1 unrotated letter & 2 unrotated letters & 3 unrotated letters \\
\hline UU & $\begin{array}{l}\text { Within-category search } \\
\text { for unrotated letter }\end{array}$ & $\begin{array}{l}\text { Between-category search } \\
\text { for unrotated letter }\end{array}$ & 1 unrotated letter & 2 unrotated letters & 3 unrotated letters \\
\hline UR & $\begin{array}{l}\text { Within-category search } \\
\text { for unrotated letter }\end{array}$ & $\begin{array}{l}\text { Between-category search } \\
\text { for unrotated letter }\end{array}$ & 1 rotated letter & 2 rotated letters & 3 rotated letters \\
\hline
\end{tabular}

Training Block 1 , all letters on every slide were of the same orientation, and thus Block 1 was considered the within-category search set. Training Block 2 trials required the subjects to detect a target letter from among letters of the opposite orientation (rotated $180^{\circ}$ ) and thus was considered between-category search. All subjects received 55 withincategory search slides during Block 1 and 55 between-category search slides during Block 2 . The order of presentation of these blocks was counterbalanced within and across groups.

Testing consisted of three blocks of trials. Block 1 contained 60 trials; Block 2, 50 trials; and Block 3, 40 trials. All testing slides contained both within- and between-category distractors. The blocking factor determined how many within-category letters were present on each slide. Testing Block 1 slides each contained one within-category letter, Block 2 two within-category letters, and Block 3 three within-category letters. The order of presentation of these blocks was counterbalanced within and across groups.

Number of distractors. Within each block of training trials, the number of distractor items per slide was varied from zero distractors to a maximum of five distractors. No slide ever contained more than six letters. Of the 55 slides within each training block, there were 5 slides with zero distractors and 10 slides each with one through five distractors, respectively. The order of presentation of the 55 slides within each block was randomized for each subject. Training Block 1 distractors were from the same category as the target, whereas Training Block 2 distractors were from the opposite category as the target.

During testing, the number of distractors referred to the number of between-category letters on each slide. Since no slide ever contained more than six letters, the maximum number of between-category distractors varied for each block. Of the 60 slides in Testing Block 1 , there were 10 slides each with zero through five between-category distractors, respectively. Of the 50 slides in Testing Block 2, there were 10 slides each with zero through four between-category distractors. Of the 40 slides in Testing Block 3, there were 10 slides each with zero through three between-category distractors. The order of presentation of the slides was randomized for each subject in each block.

Response. Slides were further subdivided within distractor levels by target present versus target absent. There were five slides at each level of distractor for which the correct response was "absent" and five for which the correct response was "present."

The testing slides each contained at least one within-category letter. During Testing Block 1, for example, $50 \%$ of the time this letter was the target letter. As in training, half of all slides at every level of betweencategory distractor contained a target letter and half did not. Response was randomized with the number of distractors for each subject within each block of both training and testing trials.

Each subject was seated in front of the tachistoscope and asked to place a blindfold over the left eye. At the beginning of each block of trials, the subject was informed of the orientation of the targets for that block. The experimenter would then place a slide in position and inform the subject to search for a particular letter. The subject initiated each trial by depressing a switch with the left hand, which in turn activated a timer. The subject's response was recorded using a two-choice (present or absent) reaction time paradigm without feedback on the cor- rectness of the response. Five-minute breaks were provided between each block of trials.

\section{RESULTS}

Since each subject received five present trials and five absent trials for each level of the number of distractors factor, the response latencies of each of these five trials were averaged and this mean was used for the purpose of analysis. Data were then subjected to a base 10 $\log (X+1)$ transformation following range procedures to determine homogeneity of variance. The Greenhouse and Geisser correction (1959) for degrees of freedom was used to reduce the probability of Type I error associated with the repeated measures design.

\section{Training Data}

Separate three-factor mixed analyses of variance with group as a between-subjects factor and block and number of distractors as within-subjects factors were performed using the present and the absent data. A significant main effect for block $[F(1,36)=6.18, p<.025]$ was found for absent responses only, with mean response latencies for Block 1 reliably slower than those for Block 2 (Tukey $H S D, p<.05$ ). Using the present data, there was a reliable block $\times$ number of distractors interaction $[F(1,36)=10.59, p<.01]$.

The main effect of number of distractors was significant for both the present $[F(1,36)=22.72, p<.001]$ and the absent data $[F(1,36)=56.29, p<.001]$. Response latency generally increased with more distractor items.

\section{Testing Data}

A three-factor mixed-design analysis was completed with group as a between-subjects factor and block and number of distractors as within-subjects factors. All three blocks and distractor levels zero through three were included in these analyses. Analyses of both the present and absent data indicated a reliable effect of block. Tukey's $H S D$ test $(p<.05)$ showed that mean response latencies for Block 3 were slower than those for Block 2, which, in turn, were slower than those for Block 1.

As can be seen from Figures 1B and 1C, the effect of number of distractors generally was to increase response 

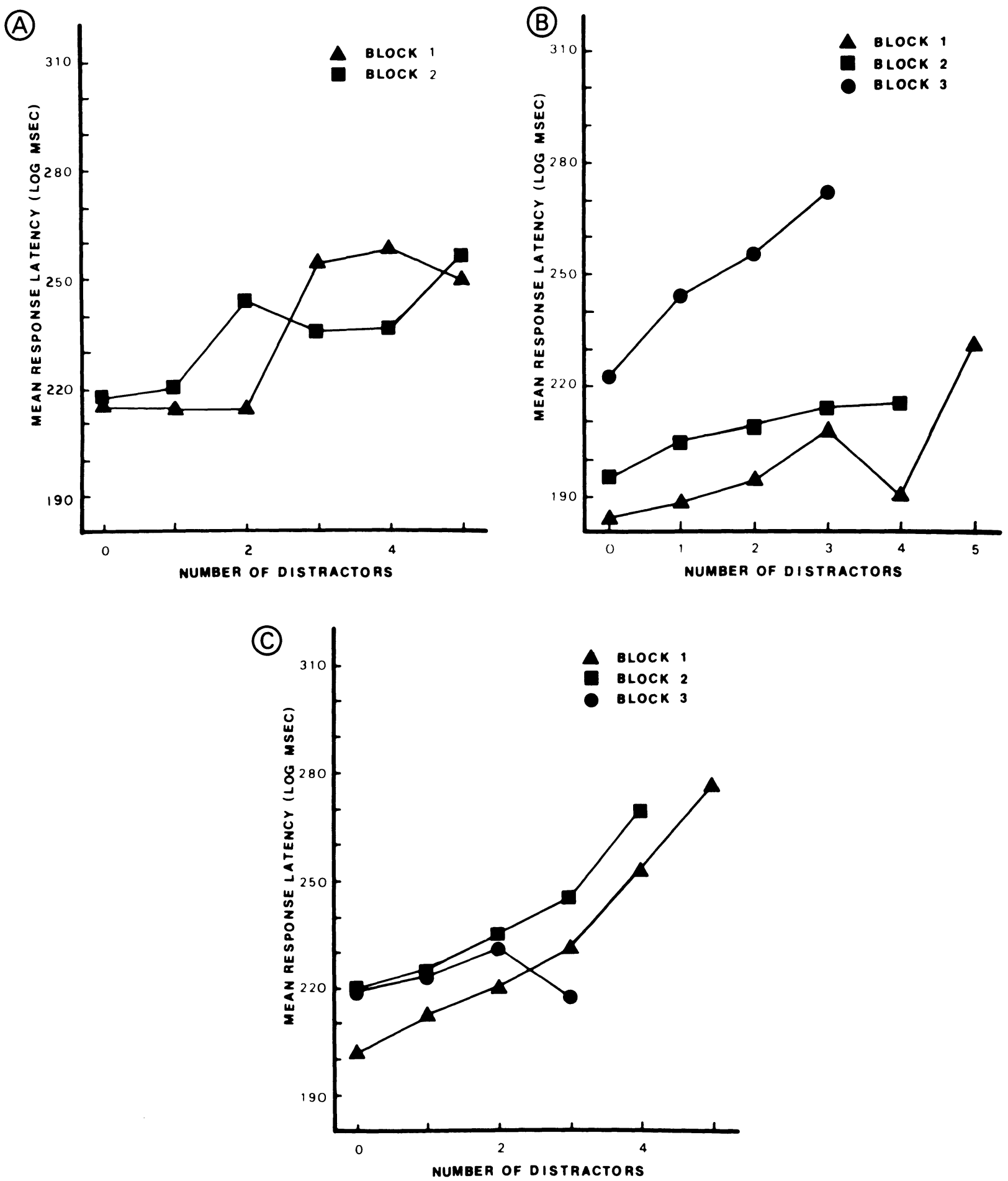

Figure 1. Mean response latency as a function of the number of distractors for each block. (A) Training present data only. (B) Testing present data only. (C) Testing absent data only.

latencies. However, this was not consistent across all blocks, as is shown by the significant block $\times$ number of distractors interaction for the present data.

It can be seen in Figures 1B and 1C that response latencies generally increase as a function of the number of distractors and the three blocks for both the present and $a b$ - sent responses. Less apparent, perhaps, is the reliable (present, Figure 1B) and marginally reliable (absent, Figure 1C) block $\times$ number of distractors interaction found in the first set of analyses. Both block and number of distractors had a strong and consistent effect on search performance in the testing trials. 


\section{Training Block 2 Versus Testing Block 1}

A major interest in this research was the comparison of performance in Training Block 2 and Testing Block 1 , since the only procedural difference between these two blocks was that Testing Block 1 required both detection and identification from the subject, whereas Training Block 2 only required detection. Hence, these two blocks were compared using a three-factor mixed analysis. Although the present data were analyzed separately from the absent data, the results were nearly identical. A significant main effect for block was found in each of the analyses $[F(1,36)=39.33, p<.001$, for present, and $F(1,36)=5.73, p<.025$ for absent]. Tukey's $H S D$ indicated that responding in Training Block 2 was significantly slower than that in Testing Block $1(p<.05)$.

A reliable effect was also found for number of distractors in both analyses $[F(1,36)=27.54, p<.001$, for present, and $F(1,36)=34.63, p<.001$, for absent $]$. The block $\times$ distractor interaction only approached significance $(p<.07)$ for the present data.

\section{DISCUSSION}

In the present study, it was predicted that sufficient practice might predispose the subject to use the category distinction when both detection and identification were required.

\section{Training}

The near-perfect selectivity found by Jonides and Gleitman (1972) was not replicated in the present study. Although the hypothesis would predict a significant effect for block (with mean response latencies being slower during Block 1), a reliable effect of block was found for absent data only. Post hoc comparisons showed that this difference was in the predicted direction; that is, within-category search latencies were slower than between-category latencies. Although a block $\times$ number of distractors interaction was predicted, it proved to be reliable only for the present data. Both the slopes for within- and between-category search as a function of the number of distractors differed from the zero point, which would have indicated perfect selectivity. However, since the slope for within-category search was greater than that for between-category search, this can be interpreted as supporting selective search during Block 2.

It should be noted that most researchers (e.g., Jonides \& Gleitman, 1972; Ingling, 1972) have used digits as opposed to letters to obtain the category effect. In the present study, we sought to minimize the physical distinctions inherent in the two categories by using the same physical stimuli for both, rotating them $180^{\circ}$ to provide distractor items. It was expected that in the present study the letter's rotation would not affect the time required to detect it. This, however, may not have been the case. A closer replication of Jonides and Gleitman (1972) in which the angularity as opposed to the curvilinearity of targets and distractors is controlled more might be used in future studies. Since, aside from horizontal orientation, the targets and distractors were physically identical, the failure to find strong evidence for the category effect may have resulted from too much homogeneity in these items.

\section{Testing}

The major goal in the testing phase was to assess whether previous experience with the category distinction (training) would carry over when both within- and between-category search was demanded on every trial.
A significant main effect for block was found, indicating that some selectivity in processing was occurring. However, the main effect for the number of between-category distractors was significant, indicating that response latencies generally slowed as the number of these distractors increased. Given the significant block $x$ distractor interaction, this slowing was not consistent across blocks. An examination of Figure 1B suggests that selectivity in processing (i.e., the ability to ignore betweencategory distractors) was occurring in Blocks 1 and 2 for up to four distractors. The consistent increases in response latencies at all levels of between-category distractors during Block 3 suggest that perhaps selectivity was no longer being used.

The slopes found in the present study support a partially selective search hypothesis. That practice increased selectivity can be argued by comparing the slopes found in the present study with those found by Francolini and Egeth (1979). For the present responses, the slopes of the functions for the one, two, and three within-category distractors found by Francolini and Egeth (1979) were 13.0, 18.7, and $14.1 \mathrm{msec} /$ distractor, respectively. In the present study, these slopes were $7.3,4.9$, and $16.1 \mathrm{msec} /$ distractor. Thus, although it cannot be argued that search was completely selective, this suggests increased selectivity as the result of the practice during the training phase of the study.

An additional manipulation that was included during testing was the group factor. Shiffrin and Schneider (1977) have argued that the category effect can be subsumed under automatic detection theory. This theory holds that, given sufficient practice with any given set of stimuli, these stimuli will come to elicit automatic responses if they are consistently mapped with these responses. If this were the case in the present study, switching the subject from upright targets to rotated targets (Group UR) and vice versa (Group $R U$ ) during testing should have eliminated any benefit gained from the training trials. Performance was not disrupted to the extent that Shiffrin and Schneider (1977) would have predicted. The role of practice in developing the category effect is not dependent on the actual items used during the task, but more on the ease with which these items can be categorized. It would appear that it is not the individual items that are being learned during the development of the category effect, but the ways in which one group of items can be distinguished from another group on the basis of their category membership.

\section{REFERENCES}

Francolini, C. M., \& Egeth, H. E. (1979). Perceptual selectivity is task dependent: The pop-out effect poops out. Perception \& Psychophysics, 25, 99-110.

Greenhouse, S. W., \& Geisser, S. (1959). On methods in the analysis of profile data. Psychometrika, 24, 95-112.

INGLING, N. W. (1972). Categorization: A mechanism for rapid information processing. Journal of Experimental Psychology, 94, 239-243.

JoNides, J., \& GleitMAN, H. (1972). A conceptual category effect in visual search: $\mathrm{O}$ as letter or as digit. Perception \& Psychophysics, $12,457-460$.

SCHNEIDER, W., \& SHIFFrin, R. M. (1977). Controlled and automatic human information processing: I. Detection, search, and attention. Psychological Review, 84, 1-66.

Shiffrin, R. M., Dumais, S. T., \& Schneider, W. (1981). Characteristics of automatism. In J. L. Long \& A. Bradley (Eds.), Attention and Performance IX. Hillsdale, NJ: Erlbaum.

ShIFfrin, R. M., \& SCHNEIDER, W. (1977). Controlled and automatic human information processing: II. Perceptual learning, automatic attending, and a general theory. Psychological Review, 84, 127-190.

(Manuscript received July 27, 1990.) 